\title{
Riesgos psicosociales que conlleva la responsabilidad del rol de tipo parental en el proyecto de vida de adolescentes hermanos mayores
}

\author{
Psychosocial risks entailed by the responsibility of the parental role in the \\ life project of older sibling adolescents
}

\author{
Jhoselyn Chávez \\ jhosy_jos@hotmail.es \\ Universidad San Francisco de Asís, Bolivia
}

\section{RESUMEN}

Este artículo presenta los aportes más significativos que realizo la autora en un trabajo de investigación, en donde se reflejan los riesgos psicosociales que conlleva la responsabilidad del rol de tipo parental en el proyecto de vida de adolescentes hermanos mayores. Esto puede verse reflejado en diferentes contextos tanto sociales, escolares como también en contextos familiares, donde los adolescentes responden de acuerdo a lo esperado para su adulto significativo. Es un estudio cuanti-cualitativo, en su parte cuantitativa es de tipo transaccional descriptivo, lo cualitativo implica la valoración de toda la data recopilada y que permite consolidar el aporte respectivo al estudio hecho. La población está constituida por 21 personas principales, la misma fue determinada por saturación de datos. Para la recolección de datos se utilizó como método el cuestionario, y como instrumento una guía de entrevista. Con la investigación realizada, se logra identificar que los hijos mayores cargan con muchas responsabilidades, por lo general desde la infancia cuidan a sus hermanos, son soporte emocional, afectando a la toma de decisiones que influyen en la familia.

Palabras clave: Riesgos socioemocionales; rol parental de los adolescente; proyecto de vida hermanos mayores

\begin{abstract}
This article presents the most significant contributions made by the author in a research work, which reflects the psychosocial risks involved in the responsibility of the parental role in the life project of adolescent older siblings. This can be reflected in different contexts, both social, school and family contexts, where adolescents respond according to what is expected for their significant adult. It is a quantitative-qualitative study, in its quantitative part it is of a descriptive transactional type, the qualitative implies the valuation of all the collected data and that allows to consolidate the respective contribution to the study made. The population is constituted by 21 main people, the same was determined by data saturation. For data collection, the questionnaire was used as a method, and as an instrument, an interview guide. With the research carried out, it is possible to identify that older children carry many responsibilities, usually from childhood they take care of their siblings, they are emotional support, affecting the decision making that influences the family.
\end{abstract}

Key words: Socio-emotional risks; parental role of adolescents; life project elder brothers 


\section{INTRODUCCIÓN}

Generalmente cuando los padres trabajan los adolescentes tienen que cuidar a los hermanos menores. Cuando ambos padres tienen empleos de tiempo completo, o una madre soltera tiene que trabajar, generalmente los adolescentes se quedan a cuidar de sus hermanos menores. Dependiendo de la estabilidad y madurez del hermano mayor, tal situación puede tener un efecto positivo o negativo en ambos niños.

Según Queliz, (2013), la sociedad legitima, el hecho de que los y las adolescentes mujeres y varones hermanos mayores de la casa se hagan cargo de los hermanos y hermanas menores y tareas del hogar "eso no es nada", es por su propio bien, para que aprenda "ella tiene que ayudarme porque soy su madre", son algunas de las excusas con las que la familia y la sociedad se dan permiso de violentar el desarrollo de esas infantas. Cuando cuidar a los hermanos y hacer los oficios de la casa se vuelve la labor central en la vida de una niña en etapa de adolescencia, entonces se limita o anula su participación en labores escolares, espacios lúdicos de diversión, imprescindibles para su desarrollo.

El tema de lo doméstico en las adolescentes pareciera que se convierte en el pasaporte para su vida. Mientras más manejan el tema, padres y madres piensan que están preparadas para la vida. Es algo forzada porque destroza muchos sueños y al observar los números de niñas y adolescentes embarazadas ya a los 13 y 14 años en el seno familiar, se identifica que la adolescente cuida o ha cuidado a sus hermanos desde pequeños $\mathrm{y}$ se supone entonces que puede cuidar de otro menor.

Según Pinto (2011) las adolescentes mujeres aprenden el sentido de la maternidad no porque quieran sino porque el entorno social en que se desenvuelven les da permiso.
Para las adolescentes es el rol que les toca jugar. La falta de educación, y específicamente de educación sexual, con énfasis en métodos adecuados de planificación a cada edad, ha acarreado la epidemia de embarazos, situación que puede revertirse con voluntad gubernamental.

El dar roles parentales a las niñas y verlas como adultas facilitas unas condiciones de relación con un adulto. "Puede ser seducida y sufrir una violación que no tiene que ser necesariamente con violencia, y en esta situación ya su persona, su desarrollo está siendo violentado. Muchas veces viene de un entorno donde ha vivido otras formas de violencia, sea exclusión, maltrato o experiencias previas de abuso sexual", indica. En otros casos, lo que sucede es que luego de una infancia al cuidado de los hermanos y la casa, la adolescente se une voluntariamente en pareja (Pinto 2011).

La realidad de los menores que cuidan a sus hermanos niños, niñas y adolescentes que son responsables de tareas cuidadoras en su medio familiar impropias para su edad. Es decir, niños, niñas y adolescentes que cuidan de sus hermanos; que toman para sí la principal responsabilidad en la ejecución de las tareas domésticas por una peculiar situación familiar; que se responsabilizan de la educación y cuidado de sus hermanos menores ante la incapacidad, indiferencia o negligencia de sus padres. Niños, niñas y adolescentes, en fin, que cargan sobre sus espaldas con tareas de atención sanitaria, doméstica y psicológica impropias para su edad.

Por su parte, Alberdi (2009) señala que, a pesar de los importantes cambios en la sociedad actual en cuanto a las nuevas formas de constitución y concepción familiar, más de la mitad de los hogares mantiene las misma estructura familiar, y que los cambios habidos 
no afecta en gran manera a las funciones familiares en sí mismas, aunque sí en la manera o en las formas y roles para llevarlas a cabo. El ejercicio de estas funciones exige modificaciones a través del tiempo, ajustándose en cada momento al crecimiento y desarrollo evolutivo de los miembros que componen el sistema familiar. La pérdida de las diferencias en el ejercicio de estas funciones crea patología familiar. No deben los padres confundirse con los hijos, ni querer ser como ellos, sino ejercer el rol de padres, es decir, ejercer estas funciones básicas con la diferencia y responsabilidad que les compete.

Por todo ello se formuló la siguiente pregunta de investigación: ¿Qué riesgos psicosociales conllevan la responsabilidad del rol de tipo parental en el proyecto de vida de adolescentes hermanos mayores? Y el Objetivo General en términos de "Determinar las características de los riesgos psicosociales que conlleva la responsabilidad del rol de tipo parental en el proyecto de vida de adolescentes hermanos mayores, del colegio adventista de Viacha".

Los Objetivos Específicos propuestos fueron: Identificar los roles y responsabilidades que los adolescentes asumen en su hogar; describir las características de los riesgos psicosociales en adolescentes que asumieron roles y responsabilidades de tipo parental; analizar de qué manera los riesgos psicosociales identificados influyen en el proyecto de vida de adolescentes hermanos mayores; y proponer un instrumento para identificar las situaciones de riesgo psicosocial que afecta en el proyecto de vida de los/las adolescentes hermanos mayores que asumen el rol de tipo parental.

\section{Referente teórico}

En el libro Escuela para Padres Don Bosco, expresa que educar no es tarea fácil y a veces saber qué papel deben asumir los hijos en la familia crea ciertas dudas. Muchos padres no saben si exigen demasiado a sus hijos o por el contrario se quedan cortos. Otros, erróneamente, esperan a que los niños sean mayores para empezar a inculcarles ciertos hábitos y normas de conducta. Desde que nacen, los niños juegan un papel muy importante en el entorno familiar. (Cataldo, 2011).

Según Rodríguez y Palacios (2008) los hermanos son muy importantes. Casi el $80 \%$ de los niños crecen con por lo menos un hermano o una hermana. Aunque puede que no se lleven bien todo el tiempo, los hermanos juegan un papel positivo en la vida de cada niño. Los hermanos y hermanas aprenden uno del otro sus primeras lecciones en cómo llevarse bien con los demás. Son amigos, compañeros de juegos y confidentes. Juntos aprenden a relacionarse con el mundo exterior. Hasta se protegen y se cuidan uno al otro.

Cuando los hermanos y hermanas no se llevan bien, el padre puede sentirse frustrado y enojado por sus peleas. Las exigencias que cada niño le impone a usted también son diferentes Es muy habitual que en la infancia la mayoría de los hermanos no se lleven bien, que se pasen la mayor parte del tiempo discutiendo y peleándose por nimiedades. (Caselli 2008).

Según Flaquer (2008) el niño dejo de ser niño, para convertirse en un adolescente, con sus propias ideas, sobre la vida, algunos padres no comprenden que les pasa a sus niños: habían sembrado margaritas y ahora recogen locotos, en las familias disfuncionales este momento puede ser un momento de mucho sufrimiento, el desprenderse entre padres e hijos duele, pero en la familia disfuncional el dolor es tan insoportable que se evita el crecimiento de los hijos. 
En algunas familias, la separación se realiza después de una pelea, en otras en cambio la separación en momento de encuentro, de contacto, de aceptación del otro, de creer en el otro, de confiar. Pero a pesar de todo duele. Todo padre debe dedicar tiempo a los hijos, un tiempo que será diario y de calidad. Se puede conocer a los hijos, se puede caminar y disfrutar juntos, sin confundir el ser amigos con ser colega, pues los padres han de marcar límites; ellos los precisan (Roudinesco, 2003).

Para Pinto (2011) la familia es un conjunto de personas que conviven bajo el mismo techo, organizadas en roles fijos (padre, madre, hermanos, etc.) con vínculos consanguíneos o no, con un modo de existencia económico y social comunes, con sentimientos afectivos que los unen, aglutinan.

Naturalmente pasa por el nacimiento, luego crecimiento, multiplicación, decadencia $\mathrm{y}$ trascendencia. A este proceso se le denomina ciclo vital de vida familiar. Tiene además una finalidad: generar nuevos individuos a la sociedad. (Pinto 2011).

Según Engelmayer (2015) uno de los aspectos más importantes es el desarrollo del conocimiento de los roles que realizan los diferentes agentes sociales. Entre ellos hay que destacar las funciones parentales, cuyo contenido es uno de los primeros en aparecer, por ser la familia el contexto de desarrollo principal y más duradero. El estudio de la atribución de funciones parentales por parte delos hijos se hace especialmente necesario por los numerosos cambios que ha sufrido la estructura y la dinámica familiar en las últimas décadas.

Así, la desestructuración familiar, o cualquier otra circunstancia en la que las funciones parentales no se ejercen de forma correcta, enfrenta a los hijos a situaciones que ponen en peligro su bienestar físico $\mathrm{y}$ emocional, afectando también a su concepto de familia. La mayoría de las investigaciones acerca de la construcción del conocimiento social se centran en estudiar cómo influye la madurez cognitiva del sujeto en la elaboración de los conceptos, hasta que se alcanzan las nociones adultas (Inhelder, y Piaget 2015).

Se coincide totalmente con Andolfi (2007) cuando señala que la estructura familiar debe asentarse en las funciones maternas y paternas. Un adecuado despliegue de las funciones parentales resulta esencial para la constitución subjetiva, y cuanto mayor sea la distancia entre ellas y su cumplimiento efectivo por parte de los progenitores, mayores serán los costos psíquicos y conflictos del niño. El padre debe sostener los significantes y los emblemas de su virilidad, y la madre los de su feminidad.

Atendiendo a lo expuesto por Minuchin, (2007) se puede entender que la presión por parte de la familia es en general el primer problema que debe afrontar una persona a lo largo de su desarrollo. Los padres suelen comunicarse con sus hijos de manera indirecta, incrementando la sensación de incertidumbre de sus hijos a la hora de tomar decisiones por sí solos; cuando alguien regala un bebé de juguete a su hija, no le está diciendo claramente "quiero que te prepares para ser madre" o "si cuando creces decides no tener hijos te convertirás en la vergüenza de la familia". Sin embargo, tampoco le expresa que no tiene la obligación de hacerlo $\mathrm{y}$ que tiene total libertad para escoger su propio camino.

\section{MÉTODO}

El enfoque que se utilizó para desarrollar la investigación que dio bases a este artículo es cuali-cuantitativo. Este método también llamado mixto, utiliza la recolección y análisis de datos para contestar la pregunta de 
investigación, confiando en la medición numérica y del conteo a partir de uso de la estadística, para establecer patrones de comportamiento de la población. Del mismo modo utiliza la descripción a partir de datos proporcionados mediante entrevistas que evidencian formas particulares de ver la realidad. Las fases de este método son la observación y la evaluación de los fenómenos de la realidad, el establecimiento de ideas, la consecuencia de las pruebas que demuestran un hecho, la revisión de estas ideas y la proporción de ideas originales. (Hernández et al 2010).

Atendiendo a lo expuesto por Tintaya (2009) la investigación responde a un tipo de investigación transaccional descriptivo que tiene como objeto indagar la incidencia de las modalidades o niveles de una o más variables en una población y tiene un diseño no experimental. Podría definirse como la investigación que se realiza sin manipular deliberadamente las variables. Es decir, se trata de estudios donde no altera en forma intencional las variables independientes para ver su efecto sobre otras variables.

La población fue clasificada, según al estrato que pertenece dentro del Sistema Educativo vigente en las Unidades Educativas de Bolivia, de esta manera la investigación se trabajó con: a) Autoridades de la Unidad Educativa, plantel administrativo, Director, profesores/as); Padres madres del curso $6^{0}$ de secundaria; Estudiantes del curso de 6으 de secundaria de la Unidad Educativa Adventista "Viacha".

La muestra fue conformada por la autoridad docentes y estudiantes que se encuentran en el Municipio de Viacha, estos fueron seleccionados a partir de la coordinación con la Unidad Educativa, por lo tanto, con base a dicha reunión e identificación, se trabajó con una muestra que cumplió las siguientes características: Director de la Unidad Educativa Adventista Viacha; equipo de docentes de secundaria; grupo de estudiantes adolescentes hermanos mayores asumen la responsabilidad del rol de tipo parental; grupo de padres y madres de secundaria de la Unidad que han dejado esta responsabilidad de tipo parental a los hijos/as mayores.

La investigación estuvo constituida por veintiún (21) personas, distribuida de la siguiente manera: un (1) director, tres (3) profesores; cuatro (4) representantes y trece estudiantes.

\section{RESULTADOS Y DISCUSIÓN}

El estudio realizado comprendió a varios actores que integran la Unidad Educativa Adventista de Viacha, así se pudo realizar cuestionarios a padres $\mathrm{y}$ madres de familia y a adolescentes. Los resultados están desglosados a continuación: 

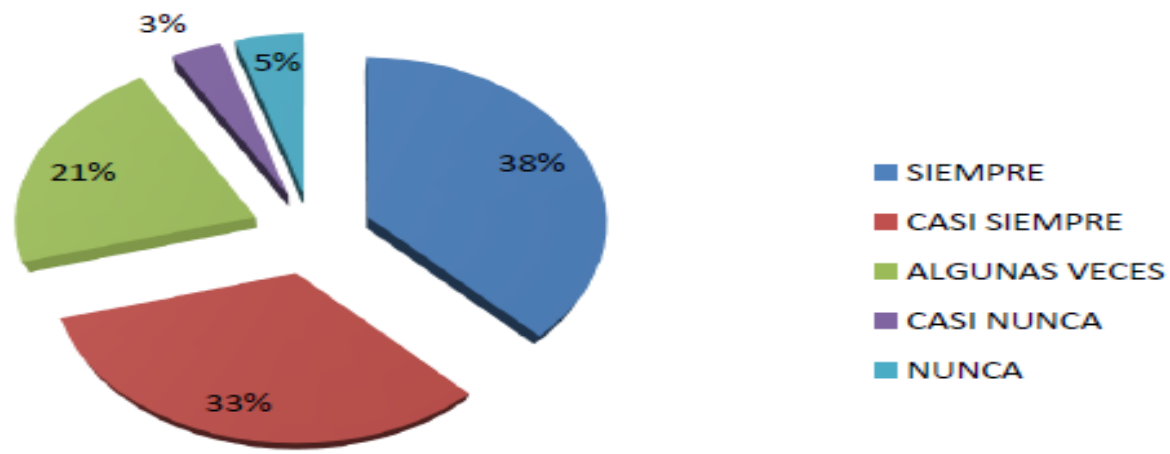

Figura 1. Porcentaje de roles y responsabilidades en el hogar

El reparto de las responsabilidades domésticas y familiares dentro del proyecto de vida en común está sujeto a un profundo proceso de cambio que afecta las formas de organización de la convivencia.

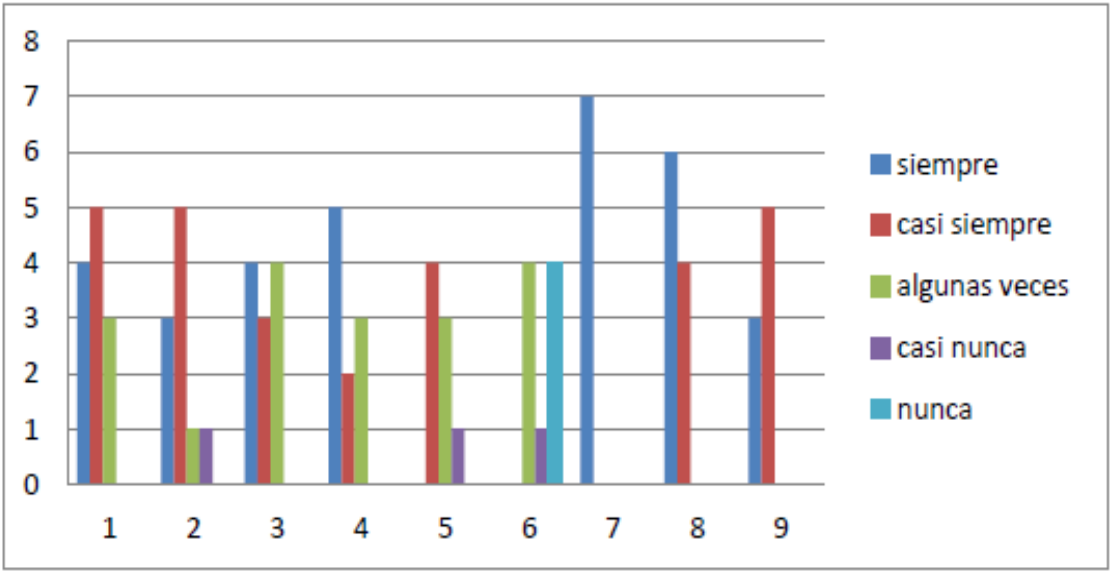

Figura 2. Convivencia en el hogar

En este cuestionario realizado para Adolescentes, sobre convivencia en el hogar que consideran en cuanto a las responsabilidades y roles en el hogar se logra identificar que en el $21 \%$ existen normas claras sobre el funcionamiento de la convivencia en la casa en cuanto a roles y comportamientos. Un $33 \%$ de la población de adolescentes considera que casi siempre ocurre peleas entre hermanos y cada hijo hace lo que quiere, cuando quiere y como quiere. Además el 38\% de la población afirma que siempre se debe colaborar en las tareas domésticas. Se identifica que $3 \%$ de los adolescentes que casi nunca cuenta con la opinión dentro de la familia para tomar decisiones. Y otro $5 \%$ considera que nunca se reúne en la semana toda la familia, al menos una vez para estar juntos $\mathrm{y}$ realizar actividades de convivencia. 


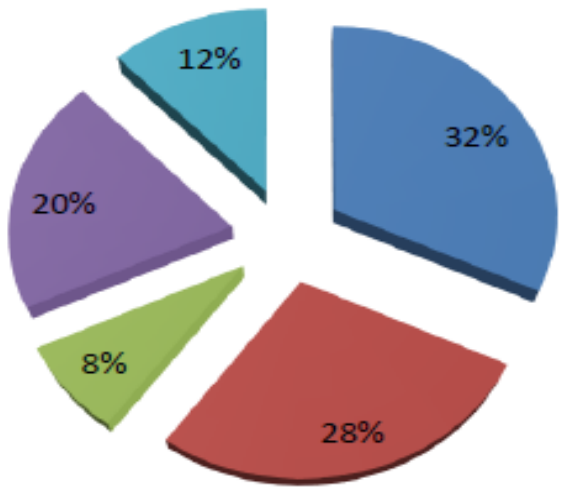

\author{
[ por problemas economicos \\ Trabajo \\ Drogadicción o alcoholismo \\ Embarazo \\ Accidente o enfermedad
}

Figura 3. Porque dejaría de estudiar

En este cuadro se observa que un $32 \%$ de los adolescentes afirman que el motivo para dejar de estudiar es por problemas económicos. Teniendo así metas académicas como el de cursar estudios a nivel técnico superior.

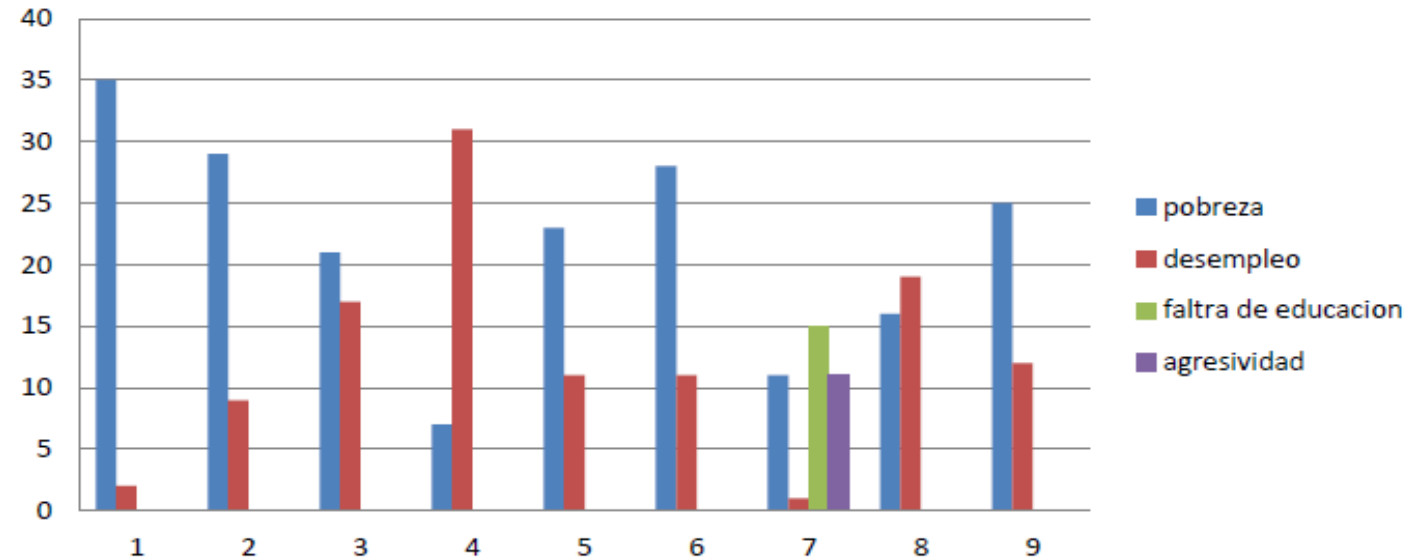

Figura 4. Causas de la violencia y delincuencia juvenil

Con un resultado alto la mayor parte considera a la delincuencia juvenil como un problema social grave en el país por la pobreza y falta de educación, el cuál necesita ser solucionado. Coinciden que él $20 \%$ han sido víctimas de un asalto y les tocó ser víctimas de un asalto. En relación con los delincuentes menores de edad creen que son culpables los padres. La principal causa que lleva al asaltante a cometer actos de violencia es la falta de educación, falta de economía y el desempleo, la edad considerada que tienen los delincuentes más frecuentes están entre los 15 - 20 años. 


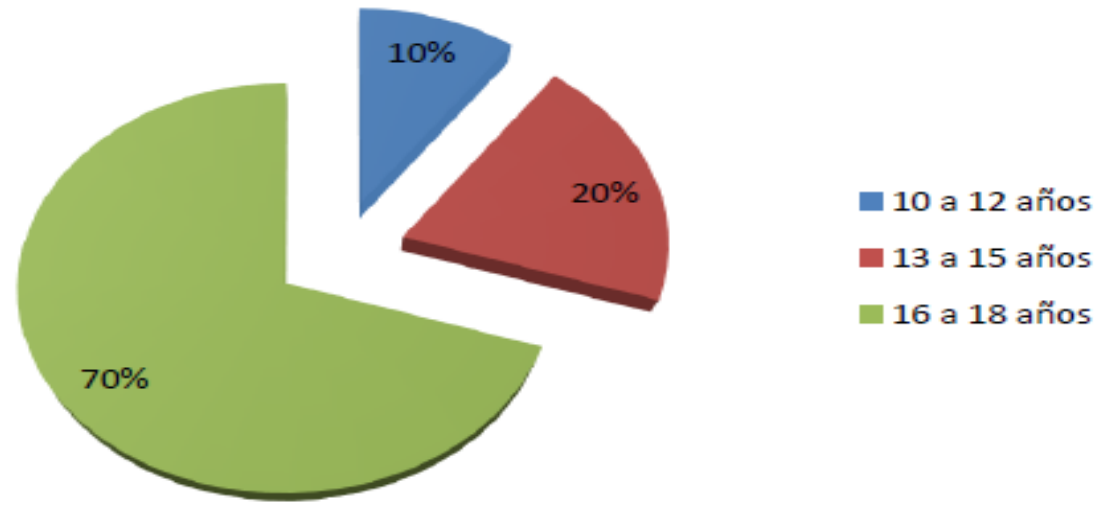

Figura 5. Embarazo Adolescente edades

En situaciones vulnerables, sin estudios y con una mala situación económica se producen embarazos con más frecuencia entre las edades de 16 y 18 años. El embarazo adolescente o a temprana edad es el que ocurre antes de los 19 años. Ser madre joven afecta a la educación. Las madres adolescentes tienen más probabilidades de abandonar la escuela secundaria. La correlación entre maternidad temprana y no completar los estudios reduce a su vez las oportunidades laborales para muchas jóvenes.

Una población no menor son las adolescentes que están entre las edades 13 a 15 años con un $20 \%$ afirma que son una población vulnerable ante el embarazo en la adolescencia.

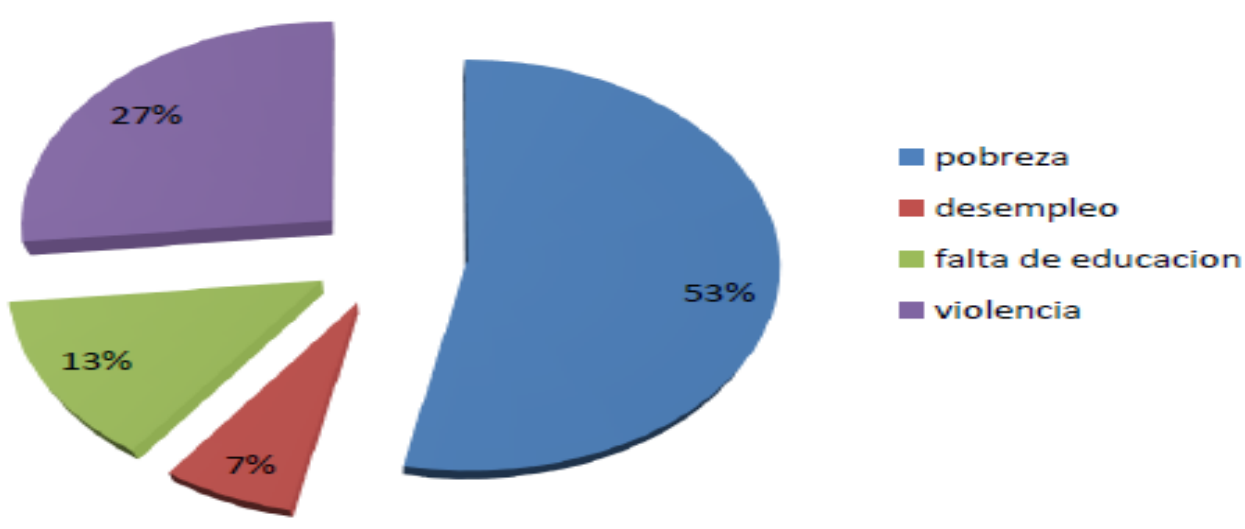

Figura 6. Causas de embarazo adolescente

Un 53\% de los adolescentes afirma que la principal causa es la pobreza. Los hijos con padres adolescentes son más propensos a pasar por la misma situación. 


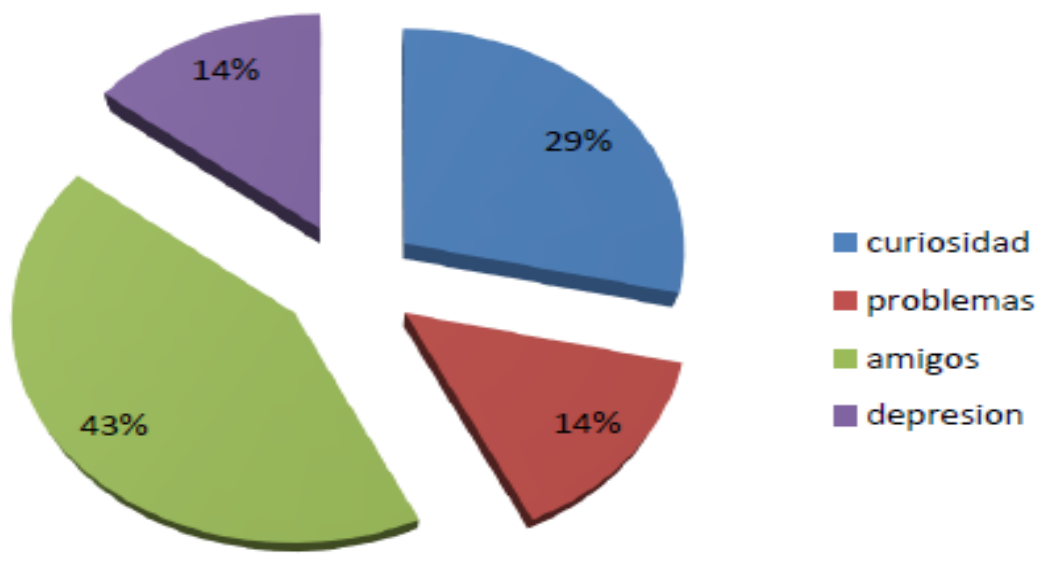

Figura 7. Motivo del Consumo de Alcohol y drogas.

El $43 \%$ de adolescente considera que opta por el consumo de alcohol y drogas la influencia de los amigos y un $29 \%$ afirma que por curiosidad llegaron a consumir alcohol y drogas. La población mayor consumidora son los jóvenes entre las edades de 15 a 16 años. El 14\% de los adolescentes consumen drogas o alcohol por problemas o depresión.

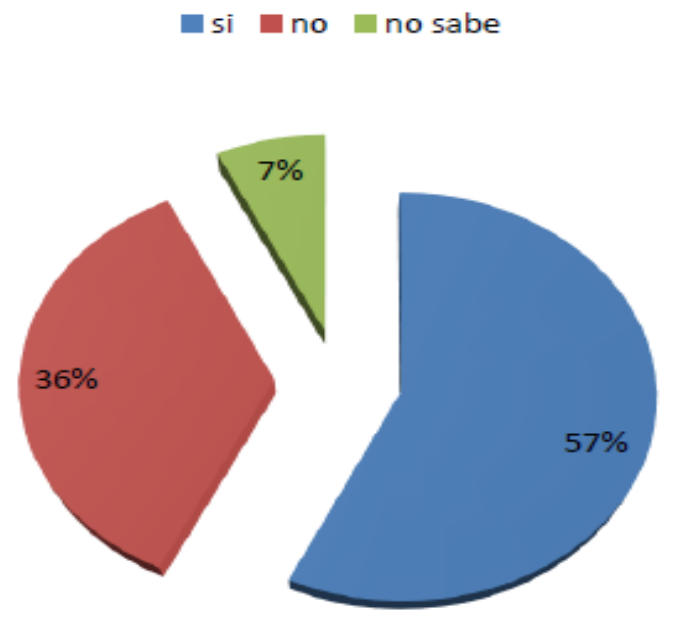

Figura 8. Cuestionario para adolescentes identificación de proyecto de vida.

Un 57\% de la población está en el proceso de planificación o tienen definido que quieren hacer después de culminar los estudios secundarios. 


\section{TECNICO $\square$ ED.SUPERIOR —POST GRADOS}

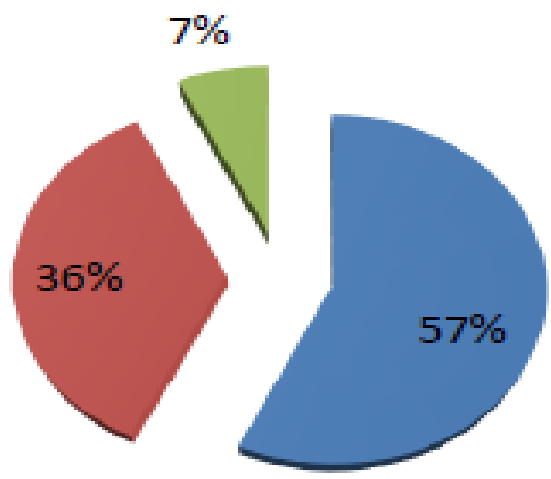

Figura 9. Metas académicas.

A través de los cuestionarios aplicados y consultados con adolescentes hermanos mayores que asumen el rol de tipo parental se logra identificar que el riesgo psicosocial grave que consideran, con un 37\% de embarazo adolescente con un 19\% deserción escolar y un 19\% también desata la delincuencia juvenil por la falta de supervisión de los padres.

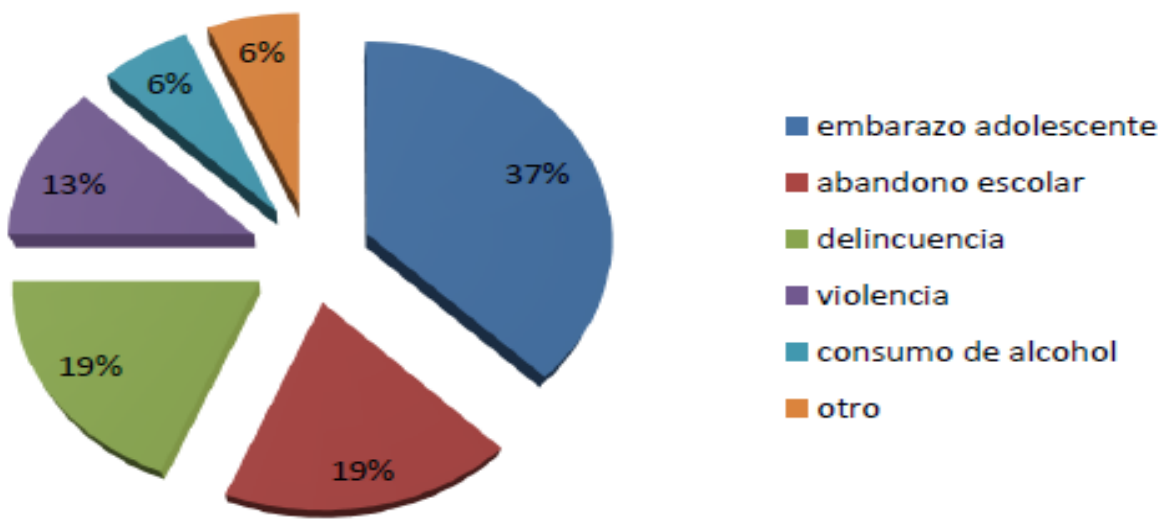

Figura 10. Identificación de riesgos sociales al asumir roles de responsabilidades de tipo parental en hermanos mayores

En un 50\% existe una relación distante e indiferente con los hijos hermanos mayores. 


\section{relacion padre -hijo/a mayor}

distante e indiferente $\square$ sobreprotectora $\square$ impositivo $\square$ permisivo

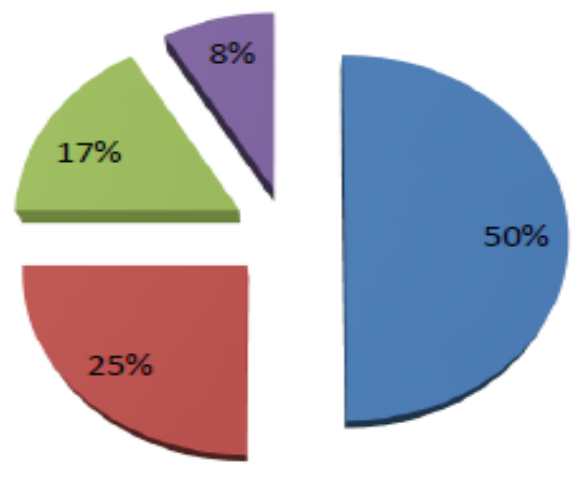

Figura 11. Cuestionario para padres y madres de adolescentes hermanos mayores

El 57\% aseguran que en cuanto salen a trabajar los padres los hijos adolescentes hermanos mayores toman el rol de cuidar de los hermanos menores y ocuparse de las actividades de la casa la relación se quiebra entre madre o padre y adolescentes.

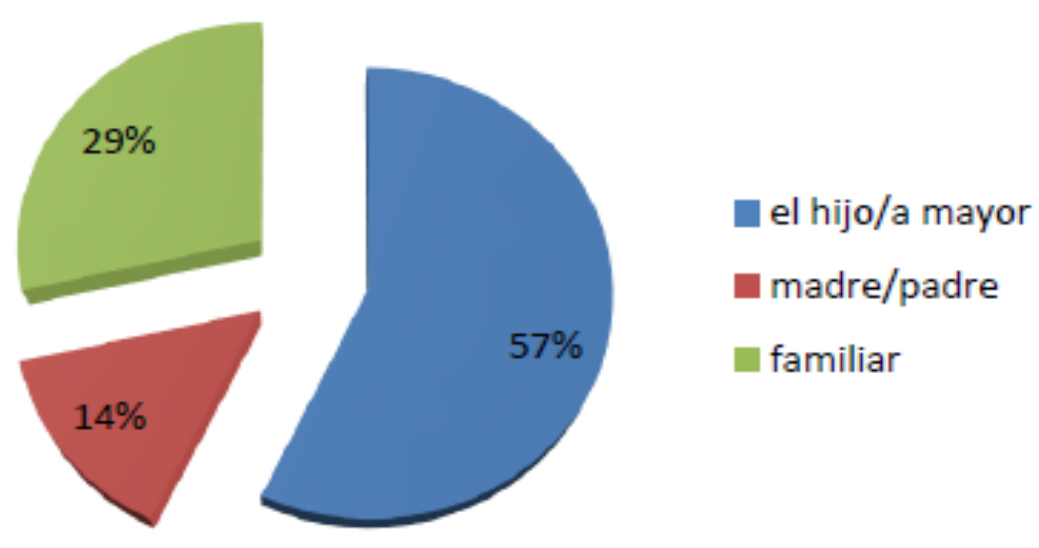

Figura 12. Quien se queda a cargo cuando sale a trabajar

El ingreso económico no logra cubrir todas las necesidades lo cual impulsa a las familias a trabajar de manera independiente. 


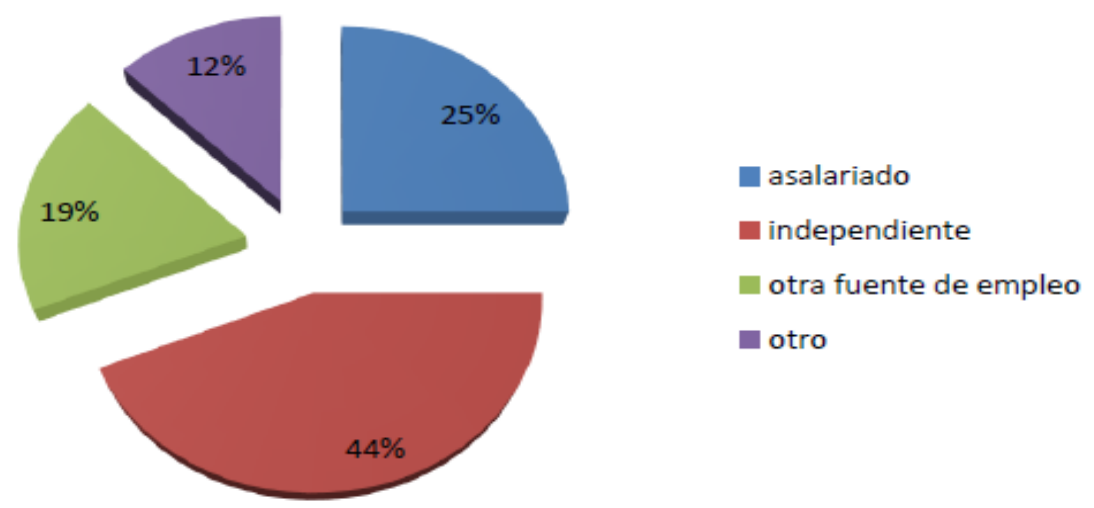

Figura 13. Ingreso económico

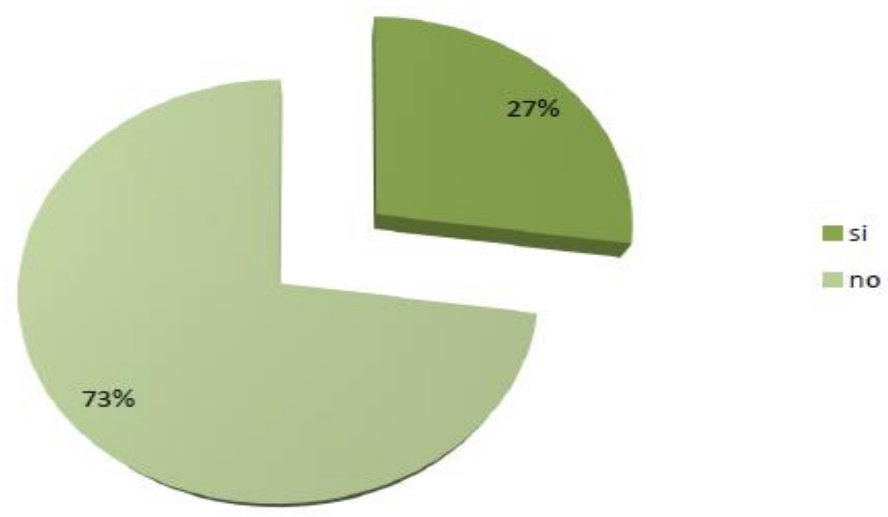

Figura 14. El ingreso económico cubre todas las necesidades

Este estudio demuestra la crisis económica que viven las familias de hijos adolescente hermano mayor en tanto el estudio revela que estas no pueden cubrir sus necesidades ya que le alcanza a la mayoría únicamente para cubrir su alimentación.

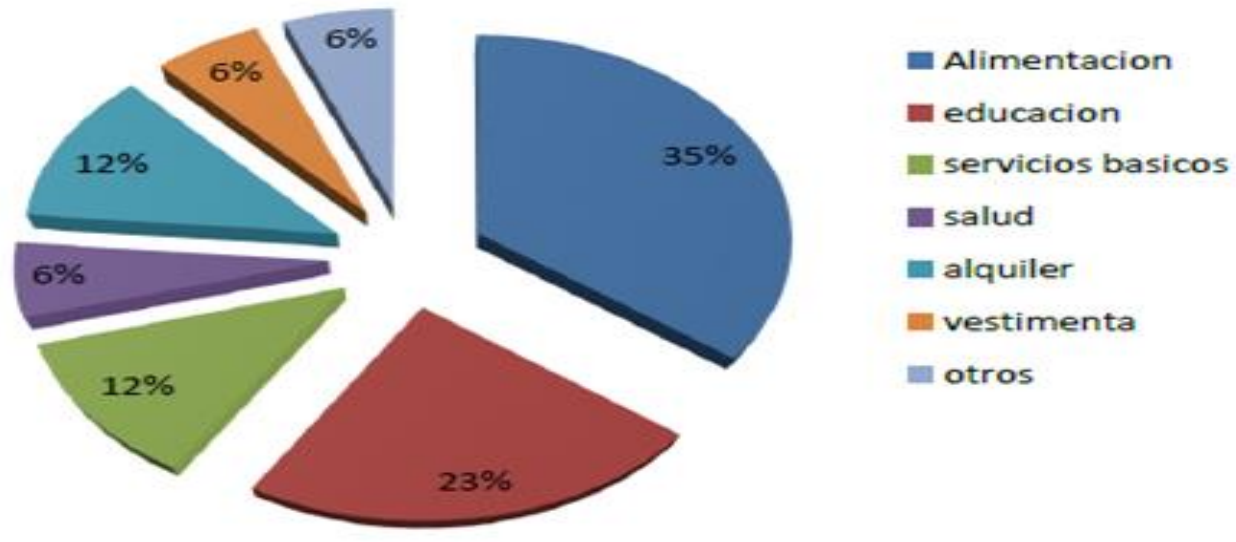

Figura 15. Gastos de los ingresos económicos 


\section{Discusión}

La familia es la base de la sociedad y en ella se pretende formar hombres y mujeres de bien con principios y valores. Las personas por naturaleza, a través del desarrollo humano han buscado el bienestar, comenzando dicho objetivo desde el mismo momento de la concepción y después reforzado sistemáticamente dentro del seno familiar, siempre y cuando éste sea sano.

Como consecuencia de la agitación y el fanatismo político en los años recientes ha ocurrido mucha violencia en el seno familiar. Desafortunadamente, el modelo clásico de familia nuclear: padre, madre e hijos que conviven juntos y afrontan la vida como una unidad, se ha venido quebrando dramáticamente en las últimas décadas.

El modelo de hogares mantenidos por el padre, con la madre en el hogar al cuidado de la crianza de sus hijos, ha quedado relegado en el pasado. Adicionalmente, el número de hogares desintegrados, se ha incrementado de manera alarmante. Con ello, los índices de madres que trabajan, se ha elevado en los últimos años, dejando la enseñanza y el rol fundamental en manos extrañas o de los mismos hijos o hijas que cumplen el rol.

La vida familiar en la comunidad, está sujeta a un profundo proceso de cambio que afecta las formas de organización de la convivencia. caracterizado, sobre todo, por la pérdida de validez de los modelos de comportamiento de los géneros y uno de los ámbitos sujetos a la negociación, en general más implícita que explícitamente, es el reparto de las responsabilidades domésticas y familiares dentro del proyecto de vida en común. (Salem 2009)

El que sea la esposa y la madre quien asuma todas las responsabilidades ha dejado de ser la norma social a seguir, no sólo porque un número creciente de mujeres se ha incorporado al trabajo asalariado, sino porque el espacio doméstico y familiar ha sido redefinido como un espacio de colaboración en un proyecto de vida en común en el que todos los miembros tienen que participar en el bienestar individual y colectivo.

La dedicación de tiempo al trabajo doméstico, sin embargo, continúa siendo muy desigual en función del sexo y la edad. Si en la infancia apenas hay diferencias ni en función de la edad, ni del género, con la entrada en la adolescencia los términos de la colaboración van cambiando mucho, tanto en función de la edad como del sexo. Las diferencias en el tiempo invertido que se apuntan en la infancia se profundizan en la adolescencia para hacerse más acusadas en la juventud, aumentando enormemente los riesgos psicosociales que conlleva esta responsabilidad.

Múltiples dimensiones y circunstancias condicionan el tiempo invertido por las personas en el trabajo doméstico. La participación de los hijos que conviven con sus padres, así como los factores que propician un reparto menos igualitario entre todos los miembros del hogar. El profundo cambio en los roles de género en lo que se refiere al trabajo remunerado no ha encontrado un cambio paralelo en lo que se refiere al trabajo no remunerado en el espacio doméstico.

El género y la edad marcan grados de participación muy diferentes, evidenciando con ello que, a pesar de los profundos cambios registrados en las pasadas décadas en los roles de género, las nuevas familias tienen problemas para socializar a las nuevas generaciones en comportamientos más igualitarios en el espacio familiar. Con la edad van diferenciándose los modelos de participación. Los hijos o hijas adolescentes colaboradores activos que asumen con cierta frecuencia además de las tareas comunes rutinaria. (Santrock, 2009). 
Por lo tanto, las recomendaciones parten a través de la investigación identificando que el papel que cada uno realiza dentro de la familia es muy importante y tiene funciones diferentes. A este papel, también se le conoce como rol. En cualquier familia, se especifican muy bien los roles que cada uno va a desempeñar, el de padre, de madre, de hijo o de hija; ejerciendo la función correspondiente a cada uno de ellos. Teniendo en cuenta que los riesgos psicosociales a los cuales son expuestos los hijos o hijas adolescentes por asumir un rol no correspondiente, como cuidar de los hermanos, asumir funciones que no le corresponden. Muchas veces por falta de tiempo de los padres u otras circunstancias se carga a los hijos mayores con responsabilidades que les lleva a adoptar un comportamiento que no es acorde con su edad y que puede afectar a su desarrollo. (Watzlawick, 2008).

Los adolescentes tienen identificado que los rodean muchos riesgos psicosociales a los cuales identifican con claridad desde la deserción escolar violencia, delincuencia juvenil, embrazo adolescente y hasta el consumo de alcohol y otras drogas, las cuales ven claramente que afecta al plan de vida que tiene reconocido la cual se teme ver alterado por los roles que deben cumplir (Dorsch 2007).

La investigación señala que los adolescentes están conscientes de los roles que forzosamente deben cubrir, lo cual lleva a establecer como metas académicas carreras técnicas con una formación de menor tiempo para contribuir con los ingresos económicos. Y postergando los estudios Superiores. Rol de padre, las funciones dentro de este rol son tan importantes como los demás, pero, requieren una mayor responsabilidad, debe proveer a la familia de protección, alimentación, cuidado, seguridad, amor, respeto, lugar donde vivir y sustento.

Uno de los roles que es el de la madre, es tan importante como el del padre, sólo que aquí se manejen mucho las emociones, el rol maternal crea un espacio especial. Para poder manejar las emociones ante cualquier situación para poder expresarlas, maneja el respeto debidamente para el comportamiento dentro y fuera de casa. También se dan casos donde puede contribuir de manera económica para el hogar.

Uno de los motivos qué más se presentan en la falta de comunicación entre padres y adolescente tiene que ver con el trabajo de los padres, que es distante e indiferente y la falta de confianza. Rol fraternal, se da entre los hijos, entre hermanos, creando complicidad $\mathrm{y}$ brindándose apoyo mutuo. Rol filial, también lo desempeñan los hijos, pero aquí su función es aprender, crecer, respetar y evolucionar como seres humanos. Las familias con pocos o muchos integrantes, forman un grupo de convivencia que necesitan reglas y estipular el desarrollo de las funciones dentro de la familia, con los roles que cada uno va a desempeñar; teniendo repercusiones favorables y positivas para el desarrollo de cada uno de los que integran la familia (Dorsch 2007).

El trabajo que se realiza dentro de una familia, debe exaltar los valores y principios que la forman para crear hombres y mujeres de bien, y puedan integrarse a una sociedad con bases sólidas que les ayudarán a forjar su futuro y formar una nueva familia, siguiendo los patrones que más han funcionado dentro de la educación que se recibió dentro del seno familiar. Asumir un rol es de manera inconsciente que día a día se va dando, claro está bajo la influencia de patrones de conducta, valores y educación que se reciban en el hogar. Ejercer de buena manera los roles 
familiares como padres, hijos, hermanos, primos, abuelos etc. conlleva a la construcción de verdaderos seres sociales, que pueden integrarse y adaptarse al mundo exterior. Un creciente significativo con la expectativa de trabajar.

\section{CONCLUSIONES}

Con relación a los padres y madres al rol que cumplen como referentes de sus hijos/as es importante, y se hace necesario que al tomar una decisión tan transcendental como la de designar un rol, deben informarse y tomar las medidas pertinentes para que los riesgos psicosociales para sus hijos/ as sean aminorados.

En el instante que se ha tomado la decisión de asignar un rol, es necesario que los progenitores puedan explicar a sus hijos/ as las razones porque se tomaran dichas acciones.

Se debe contar con asesoramiento profesional cuando existan dudas a la hora de educar, o frente a la relación que mantienen como pareja. Existen varias instituciones, que brinde seguridad y calidez, para formar mejores personas.

Se considera asumir la responsabilidad de cuidar a hijos/as que no son hijos/as propios, deben tomar en cuenta sus capacidades, para saber sus dificultades y modificarlas en bien de todo el grupo.

La relación que mantenga con las personas a su cargo debe ser cálida, es decir llena de expresiones afectivas positivas, para fomentar la seguridad de estas personas que se sienten desamparadas.

Al respecto es necesario tener presente un diagnóstico social, el cual permitirá a la Unidad Educativa hacer frente a las situaciones emergentes que provoca la realidad socioeconómica de los/las adolescentes que asisten a sus aulas. De esta manera, la escuela estaría a la vanguardia para cuidar y proteger el capital humano en sus manos.

Recordar que las Unidades Educativas, forman de manera integral a los/las estudiantes y por tanto las respuestas deben ser holístico. Para tal tarea es necesario coordinar con las instancias estatales llamadas por la ley para responder oportunamente $y$ efectivamente a las necesidades del educando.

Este estudio, se constituye en un diagnóstico inicial, para poder responder de manera inmediata a las necesidades de los estudiantes que cumplen un rol de mucho peso para los adolescentes.

Poner disposición todo el aparato burocrático dedicado a la niñez y adolescencia a elaborar leyes y normas para que las familias reciban el cuidado $y$ protección que se merecen, cuidado el capital humano de nuestro país, pero además en retribución ala enorme contribución económica que hacen estas personas al estado con su trabajo.

\section{REFERENCIAS}

Alberdi, I. (2009). La nueva familia española. Madrid: Taurus.

Andolfi, M. (2007). Terapia familiar. Barcelona. Paidos

Cataldo, CH.Z. (20111). Aprendiendo a ser padres. Conceptos y contenidos para el diseño de programas de formación de padres. Madrid editorial Visor

Caselli, M. (2008). Matrimonio en crisis. Editorial: SB. Buenos Aires Argentina

Dorsch (2007). Ambiente psicosocial y salud. Desencadenantes del estrés laboral. Madrid: Eudema

Engelmayer, 0. (2015) Psicologia Evolutiva de la Infancia y de la Adolescencia. Buenos Aires. Editorial Kapelusz

Flaquer, LL. (2008). El destino de la familia. Barcelona: Ariel.

Hernández, Fernández y Baptista. (2010) Metodología de la investigación ( $3^{\circ}$ ed). Mexico: Mc Graw-Hill 
Inhelder, B., y Piaget, J. (2015). De la lógica del niño a la lógica del adolescente. Barcelona: Paidós

Minuchin, S. (2007). Familias y Terapia familiar. Barcelona: Granica.

Pinto, A. (2011). Sistema de Evaluación de Niños y Adolescentes. Manual de aplicación, corrección e interpretación. Madrid: TEA Ediciones

Queliz Martha (2013) niños que cuidad niños. Listín Diario Santo Domingo

Rodríguez, M.J. y Palacios, J. (20088). "Familia y desarrollo humano". Madrid: Alianza Editorial
Roudinesco, E. (2003) La familia en desorden, Buenos Aires, Fondo de Cultura Económica

Santrock, J.W. (2009). Psicología del desarrollo en la adolescencia. Madrid: McGraw-Hill

Salem G. (2009). Abordaje terapéutico de la familia. Barcelona: Masson

Tintaya, P. (2009). Metodología de la investigación. La Paz, Bolivia: Gráfica Singular

Watzlawick, P. (2008). El cambio familiar: desarrollo y modelos. Buenos Aires: Gedisa 